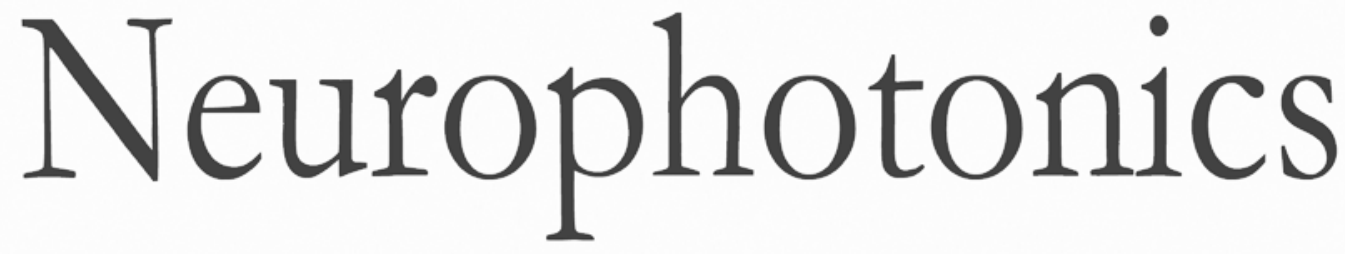

\title{
Nanoscale organization of synaptic adhesion proteins revealed by single- molecule localization microscopy
}

Ingrid Chamma

Florian Levet

Jean-Baptiste Sibarita

Matthieu Sainlos

Olivier Thoumine 


\title{
Nanoscale organization of synaptic adhesion proteins revealed by single-molecule localization microscopy
}

\author{
Ingrid Chamma, ${ }^{a, b}$ Florian Levet, ${ }^{a, b, c, d, e}$ Jean-Baptiste Sibarita, ${ }^{a, b}$ Matthieu Sainlos, ${ }^{a, b}$ and Olivier Thoumine ${ }^{a, b, *}$ \\ ${ }^{a}$ Centre National de la Recherche Scientifique, Interdisciplinary Institute for Neuroscience, UMR 5297, 147 rue Léo-Saignat, \\ Bordeaux Cedex 33077, France \\ bUniversity of Bordeaux, Interdisciplinary Institute for Neuroscience, 147 rue Léo-Saignat, Bordeaux Cedex 33077, France \\ ${ }^{c}$ Centre National de la Recherche Scientifique, Bordeaux Imaging Center, UMS 3420, Bordeaux 33077, France \\ dUniversity of Bordeaux, Bordeaux Imaging Center, Bordeaux 33077, France \\ eInstitut National de la Santé et de la Recherche Médicale, Bordeaux Imaging Center, US 04, Bordeaux 33077, France
}

\begin{abstract}
The advent of superresolution imaging has created a strong need for both optimized labeling strategies and analysis methods to probe the nanoscale organization of complex biological structures. We present a thorough description of the distribution of synaptic adhesion proteins at the nanoscopic scale, namely presynaptic neurexin-1 $\beta$ (Nrx1 $\beta)$, and its two postsynaptic binding partners neuroligin-1 (Nlg1) and leucine-richrepeat transmembrane protein 2 (LRRTM2). We monitored these proteins in the membrane of neurons by direct stochastic optical reconstruction microscopy, after live surface labeling with Alexa647-conjugated monomeric streptavidin. The small probe $(\sim 3 \mathrm{~nm})$ efficiently penetrates into crowded synaptic junctions and reduces the distance to target. We quantified the organization of the single-molecule localization data using a tesselation-based analysis technique. We show that Nlg1 exhibits a fairly disperse organization within dendritic spines, while LRRTM2 is organized in compact domains, and Nrx1 $\beta$ in presynaptic terminals displays a dual-organization pattern intermediate between that of $\mathrm{Nlg} 1$ and LRRTM2. These results suggest that part of Nrx $1 \beta$ interacts transsynaptically with NIg1 and the other part with LRRTM2. ๑ 2016 Society of Photo-Optical Instrumentation Engineers (SPIE) [DOI: 10.1117/1.NPh.3.4.041810]
\end{abstract}

Keywords: superresolution; synapse; adhesion proteins; direct stochastic optical reconstruction microscopy; SR-Tesseler.

Paper 16046SSR received Jul. 4, 2016; accepted for publication Oct. 11, 2016; published online Nov. 3, 2016.

\section{Introduction}

Synaptic contacts are dynamic macromolecular platforms with extreme spatial and temporal coordination allowing information transfer between neurons. The efficiency of synaptic transmission relies in part on a high level of compartmentation. Indeed, synapses are composed of several subdomains with very high local protein concentration including the cytomatrix active zone, the synaptic cleft, and the postsynaptic density. Single-moleculebased localization microscopy techniques, including photoactivation localization microscopy (PALM), ${ }^{1,2}$ stochastic optical reconstruction microscopy (STORM), ${ }^{3,4}$ and point accumulation for imaging in nanoscale topography (PAINT), ${ }^{5,6}$ have provided high-resolution maps of synaptic protein localization, allowing a better understanding of the organization of these submicron multisubunit assemblies. ${ }^{7-12}$ Adhesion molecules such as neurexins (Nrxs) and its binding partners neuroligins (Nlgs) and leucine-rich repeat transmembrane proteins (LRRTMs) are important actors at synapses, not only maintaining a physical contact between pre- and postsynaptic membranes, but also participating in synapse formation, differentiation, and activity. ${ }^{13-15}$

In the absence of high-quality antibodies for surface labeling of Nlgs and LRRTMs, we recently developed a method based on streptavidin monomers (mSA) ${ }^{16}$ to deliver bright organic fluorophores to proteins that are enzymatically biotinylated on a

*Address all correspondence to: Olivier Thoumine, E-mail: olivier.thoumine@ u-bordeaux.fr
15 amino acid acceptor peptide (AP) tag. ${ }^{17}$ We used this technique to label neuroligin-1 (Nlg1) and leucine-rich-repeat transmembrane protein 2 (LRRTM2) at postsynapses by direct STORM (dSTORM) and demonstrated the different nanoscale organization of these two proteins, which may underlie divergent physiological roles at the synapse. ${ }^{18}$ In addition, we and others previously showed by using single-particle tracking that neurexin-1 $\beta(\operatorname{Nrx} 1 \beta)$ diffuses relatively quickly in the axonal membrane and accumulates transiently at presynapses, ${ }^{18,19}$ but the precise nanoscale organization of $\operatorname{Nrx} 1 \beta$ within presynaptic terminals remains undescribed. However, deciphering the spatial distribution of Nrx is important to understand the interplay between the presynaptic release machinery and synaptic cleft components, especially given the dramatic impact of Nrx knock-out on synaptic transmission. ${ }^{20}$ Furthermore, given that $\operatorname{Nrx} 1 \beta$ can bind to $\mathrm{Nlg} 1$ and LRRTM2 in a competitive manner and with similar affinity, ${ }^{21-23}$ it would be interesting to know if $\operatorname{Nrx} 1 \beta$ has a preferential transsynaptic interacting partner.

In this paper, we use the mSA strategy to label $\operatorname{Nrx} 1 \beta$ molecules at presynapses in combination with dSTORM and SRTesseler, a tessellation-based analysis technique, ${ }^{24}$ to quantify the local protein organization of $\mathrm{Nrx} 1 \beta$, Nlg1, and LRRTM2 within synapses. We show that Nlg1 exhibits a rather disperse organization in dendritic spines, while LRRTM2 is organized in

$2329-423 X / 2016 / \$ 25.00$ (C) 2016 SPIE 
compact and highly enriched domains. Interestingly, $\mathrm{Nrx} 1 \beta$ in presynaptic terminals displays both a diffuse organization resembling that of $\mathrm{Nlg} 1$ and well-defined clusters reminiscent of the LRRTM2 organization, suggesting that $\operatorname{Nrx} 1 \beta$ interacts independently with both partners at synapses.

\section{Results}

\subsection{Nanoscale Organization of $N r \times 1 \beta$ at Presynapses}

To image Nrx at presynapses, primary rat hippocampal neurons were electroporated at the time of plating (day in vitro DIV 0) with a $\operatorname{Nrx} 1 \beta$ construct carrying a 15 amino acid $N$-terminal biotin acceptor peptide (AP) tag (AP-Nrx1 $\beta$ ), together with the endoplasmic reticulum-resident biotin ligase $\left(\mathrm{BirA}^{\mathrm{ER}}\right),{ }^{17}$ and a VGlut1-Super Ecliptic (SEP) presynaptic reporter. Biotin naturally present in the culture medium is covalently added to a central lysine residue in the AP-tag through the enzymatic action of the biotin ligase, and $\mathrm{AP}-\mathrm{Nrx} 1 \beta$ reaches the plasma membrane in a biotinylated form. ${ }^{25}$ The biotinylated (a)
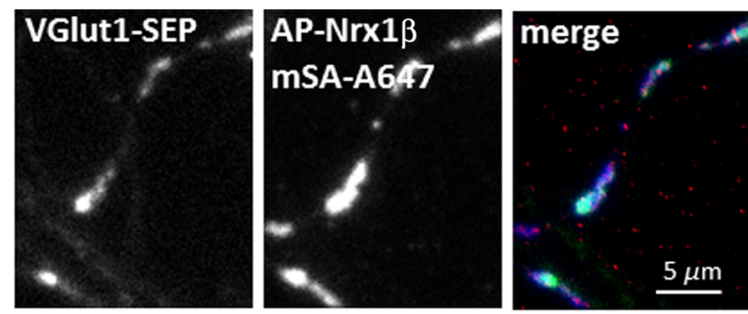

(b)
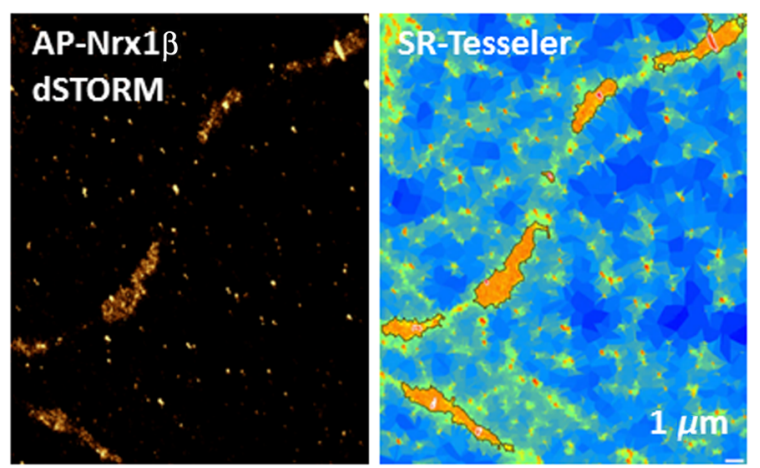

(c)

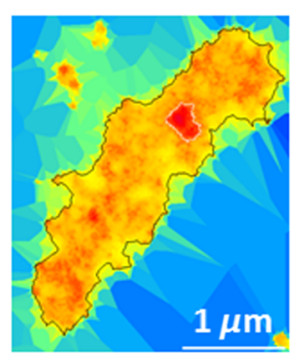

(d)

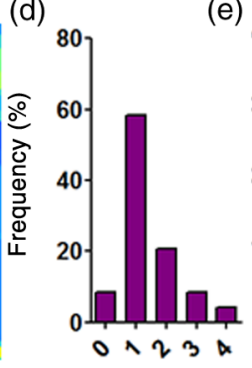

(e)

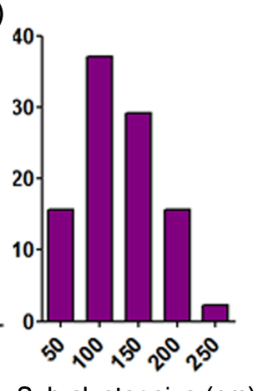

Sub-cluster \# Sub-cluster size (nm)

Fig. 1 Nanoscale organization of $\mathrm{Nrx} 1 \beta$ at the presynapse. (a) DIV 15 neurons expressing the presynaptic marker VGlut1-SEP, AP-Nrx1 $\beta$, and BirA ${ }^{\mathrm{ER}}$ were labeled with mSA-Alexa647 and fixed for dSTORM. (b) dSTORM image of an axonal segment with four presynapses and corresponding Voronoï diagram showing $\mathrm{Nrx} 1 \beta$ enrichment at presynapses, as well as subcluster formation within these enriched regions. (c) Zoom on an Nrx1 $\beta$ subcluster in the polygon representation. (d) Number of $\mathrm{Nrx} 1 \beta$ subclusters per presynapse. (e) Histogram showing the sizes of $\operatorname{Nrx} 1 \beta$ subclusters. tag is then detected by incubation with monomeric streptavidin (mSA) conjugated to an organic dye (Alexa647), compatible with dSTORM. In DIV 15 neurons, we observed an excellent colocalization of the Alexa647 mSA conjugate with VGlut1SEP [Fig. 1(a)], indicating that mSA-labeled AP-Nrx1 $\beta$ efficiently reaches presynapses. The axonal shaft was almost depleted of staining, probably owing to the fast diffusion of Nrx $1 \beta$ in this compartment. ${ }^{18,19}$ Zooming on presynapses to decipher the internal substructure of $\operatorname{Nrx} 1 \beta$ distribution, we found that $\operatorname{Nrx} 1 \beta$ exhibits a dual-localization pattern: one population fairly dispersed within the presynaptic membrane and a second population condensed into 1 to 2 nanocluster(s) (mean $1.4 \pm 0.18,24$ synapses) with higher protein density [Figs. 1(b), 1(c), 1(d)]. Using the SR-Tesseler segmentation technique, ${ }^{24}$ we determined the size of those nanoclusters, which lays in the range of 50 to $200 \mathrm{~nm}$ (median $118 \mathrm{~nm} \pm$ IQR 80 to $-154 \mathrm{~nm}$, 37 synapses) [Fig. 1(e)]. These nanoclusters match the size of confinement domains previously observed by uPAINT performed on green fluorescent protein (GFP)-Nrx1 $\beta$ labeled with Atto647N-conjugated anti-GFP nanobody, ${ }^{18}$ which most likely correspond to $\operatorname{Nrx} 1 \beta$ being trapped at presynapses.

\subsection{Difference of Organization Between Nrx1 $\beta$, Nlg1, and LRRTM2 at Synapses}

To gain insight on the nanoscale organization of postsynaptic adhesion proteins, we imaged Nlg1 and LRRTM2 by dSTORM in DIV 15 neurons (Fig. 2). Nlg1 was both present in the shaft and mildly enriched in dendritic spines, where it exhibited a fairly homogeneous distribution [Fig. 2(a)]. In contrast, LRRTM2 was almost depleted from the shaft and highly enriched in dendritic spines, where it formed one main central cluster with higher protein density [Fig. 2(b)]. To compare the nanoscale organization of Nlg1 and LRRTM2, we used SR-Tesseler ${ }^{24}$ to define a local molecular density parameter $d$ expressed in a log scale. For Nlg1, the distribution of molecule density exhibited a single population [Fig. 2(d)], reflecting the fact that Nlg1 covers a continuum of organizational states, from a very diffuse state in the shaft to the formation of small synaptic and extrasynaptic aggregates. ${ }^{18}$ In contrast, the $d$ distribution for LRRTM2 was broader and composed of two populations [Fig. 2(e)]: a first population of lower density revealing a relative depletion of LRRTM2 from the shaft compared to synapses and a second population of much higher protein density, likely corresponding to the highly enriched synaptic LRRTM2 domains. ${ }^{18}$ Interestingly, when applying the same method to presynapses, $\operatorname{Nrx} 1 \beta$ also showed a bimodal $d$ distribution [Fig. 2(f)], with a first large peak of lower density likely representing $\operatorname{Nrx} 1 \beta$ present in the axon shaft and at presynapses, and a second peak much shifted to the right, matching the enriched protein density in nanoclusters [Fig. 2(c)]. Altogether, these results suggest that $\operatorname{Nrx} 1 \beta$ displays two modes of organization: a diffuse state resembling Nlg1 organization and a concentrated state reminiscent of LRRMT2 or even more enriched.

\subsection{Nrx1 $\beta$ Organizational Patterns Display Close Similarities to Both NIg1 and LRRTM2}

To compare the nanoscale organization of presynaptic $\operatorname{Nrx} 1 \beta$ facing that of postsynaptic Nlg1 and LRRTM2, we defined the enrichment factor $R$, which measures the normalized molecular concentration on a linear scale. This enrichment factor was then 
(a)

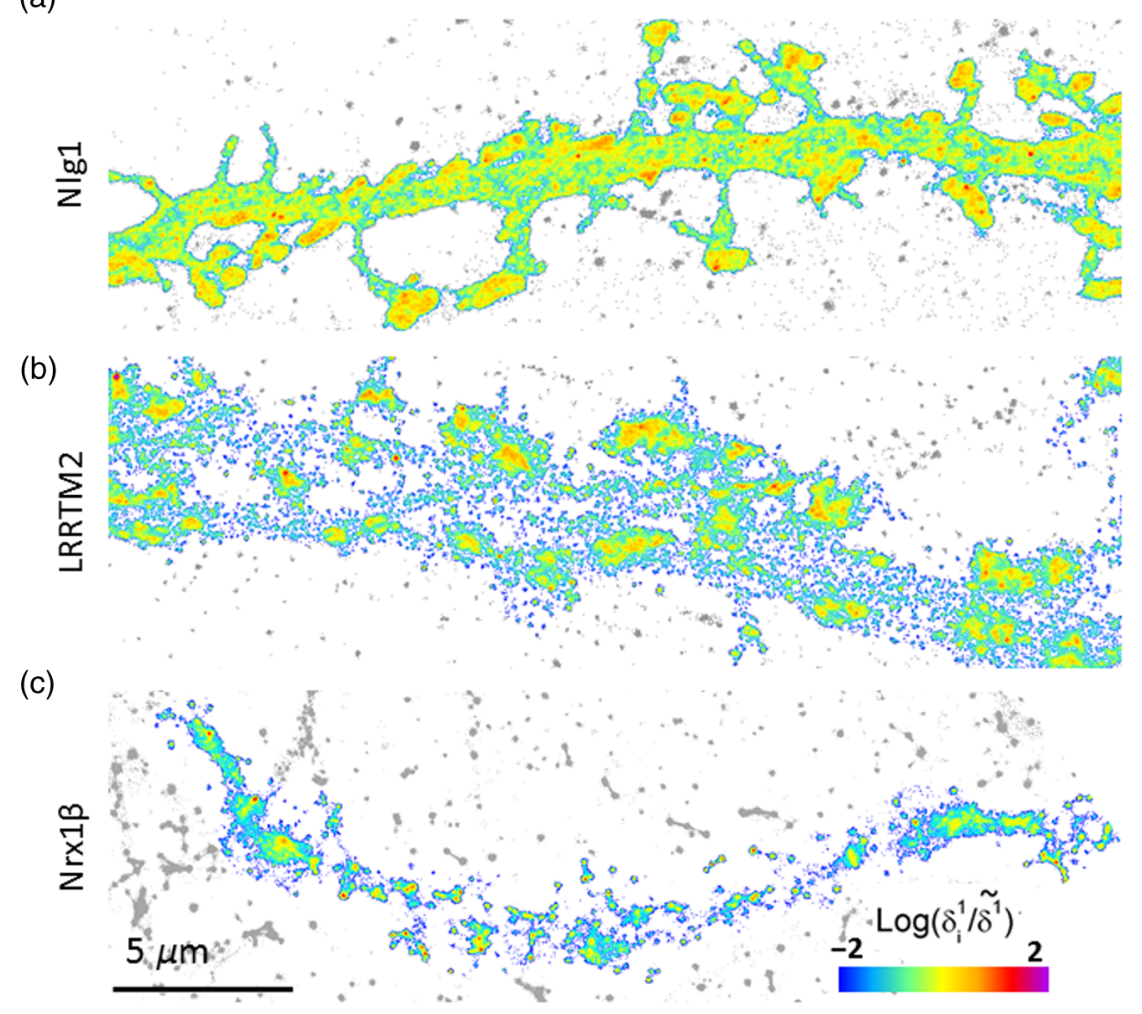

(d)

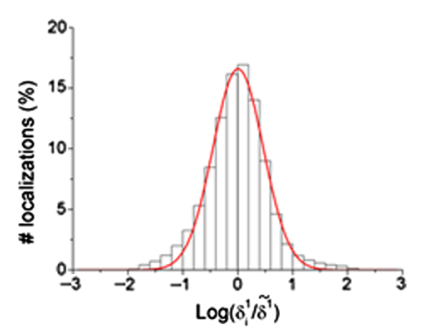

(e)

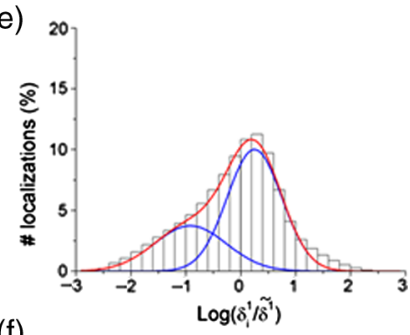

(f)

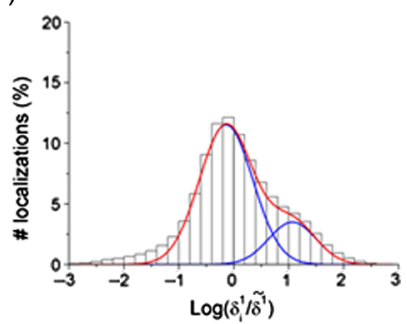

Fig. 2 Differential organization of Nrx1 $\beta$, Nlg1, and LRRTM2 in synaptic compartments. (a, c, and e) Color-coded images showing the normalized molecular densities in log scale for (a) Nig1, (b) LRRTM2, and (c) Nrx1 $\beta$ obtained from dSTORM images of AP-tagged proteins labeled with mSA-Alexa647. Note the relatively homogenous localization of Nlg1 (green and yellow values) throughout the shaft and dendritic spines, as compared to Nrx1 $\beta$ and LRRTM2 which are highly segregated in pre- and postsynaptic compartments, respectively (blue in the shaft and red in synapses). (d, e, and f) Corresponding distributions of the relative molecular density in semilog plot for Nlg1, LRRTM2, and Nrx1 $\beta$. Statistics: Nlg1 (3 cells, 3,653,239 localizations, $R^{2}=0,998$ ), LRRTM2 (5 cells, 2,971,150 localizations, $\left.R^{2}=0,992\right)$, and Nrx1 $\beta$ (5 cells, $1,582,629$ localizations, $\left.R^{2}=0,988\right)$. Note the single population for Nlg1, and the dual populations for LRRTM2 and Nrx1 $\beta$.

used as a threshold to highlight the enriched areas with respect to nonenriched ones [Fig. 3(a)].

We then graphed two parameters as a function of $R$ : the fraction of neuronal area occupied by enriched regions $A(R)$ [Fig. 3(b)] and the fraction of the number of localizations inside those enriched regions $N(R)$ [Fig. 3(c)]. Interestingly, $A(R)$ was significantly lower for LRRTM2 than for Nlg1, confirming that LRRTM2 is segregated in smaller regions in the spines than Nlg1. When $A(R)$ becomes equivalent for Nlg1 and LRRTM2 for higher $R$ [Fig. 3(b)], $N(R)$ remains two- to threefold higher for LRRTM2 compared to Nlg1 [Fig. 3(c)], indicating that these LRRTM2 segregated regions are much denser.

On the other hand, the curve $A(R)$ for $\operatorname{Nrx} 1 \beta$ is situated between those of Nlg1 and LRRTM2. $A(R)$ falls deeper than the curve corresponding to $\mathrm{Nlg} 1$ due to the fact that the diffuse $\operatorname{Nrx} 1 \beta$ population covers a relatively small presynaptic surface area compared to $\mathrm{Nlg} 1$. In parallel, the curve $A(R)$ for $\mathrm{Nrx} 1 \beta$ remains higher than that of LRRTM2, because LRRTM2 is segregated in small regions in the spines and lacks a diffuse population. Finally, while $\operatorname{Nrx} 1 \beta$ is slightly more concentrated than LRRTM2 for low $R, N(R)$ becomes equivalent for large $R$. This suggests that $\operatorname{Nrx} 1 \beta$ has a dual organization, one part being fairly diffuse and disappearing when reaching intermediate $R$ and the other more concentrated with a magnitude equivalent to LRRTM2.

\section{Discussion}

Using quantitative localization-based superresolution microscopy, we unraveled for the first time the relative distribution of presynaptic $\operatorname{Nrx} 1 \beta$ and its postsynaptic binding partners $\mathrm{Nlg} 1$ and LRRTM2 in primary hippocampal neurons. We implemented a pipeline combining high density labeling with small photo-robust monomeric probes, allowing accurate localization in synaptic compartments, with a dedicated polygonbased analysis of single-molecule localizations distribution obtained by dSTORM. This analysis allowed the calculation of protein concentration gradients, going much beyond traditional approaches based on intensity line scans to determine protein enrichment. ${ }^{24}$ We demonstrate that $\operatorname{Nrx} 1 \beta$ exhibits a dual distribution, comprising a diffuse presynaptic component that matches the distribution of postsynaptic Nlg1, plus 1 to 2 compact nanoclusters enriched in a similar way compared to LRRTM2 domains. This suggests that one population of $\operatorname{Nrx} 1 \beta$ molecules may diffuse alone or could be transsynaptically linked to $\mathrm{Nlg} 1$ as small molecular complexes, while another population of $\operatorname{Nrx1} \beta$ may be aggregated in connection to large LRRTM 2 domains. The fact that $\operatorname{Nrx} 1 \beta$ cannot simultaneously bind Nlg1 and LRRTM2 $2^{21,26}$ suggests that these two types of distributions are mutually exclusive. Based on our previous dual-color uPAINT experiments on $\operatorname{Nrx} 1 \beta$ versus $\mathrm{Nlg} 1$ or 
(a)

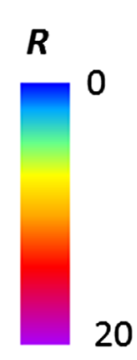

Nlg1

20

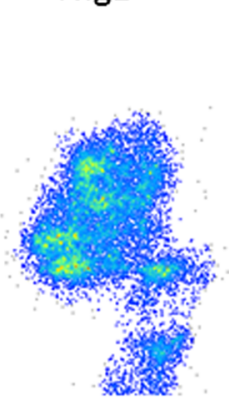

LRRTM2

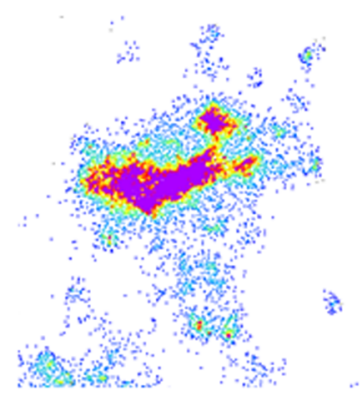

Nrx13

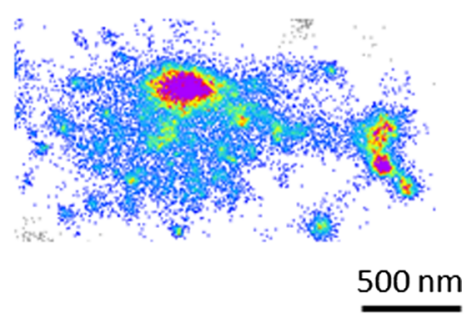

(b)

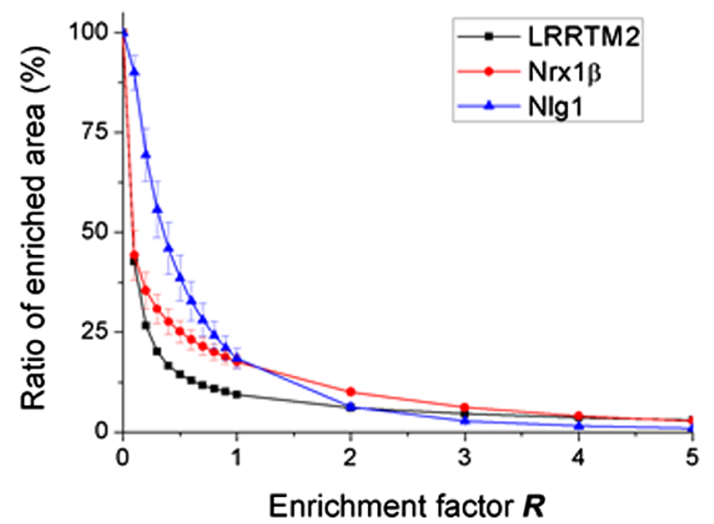

(c)

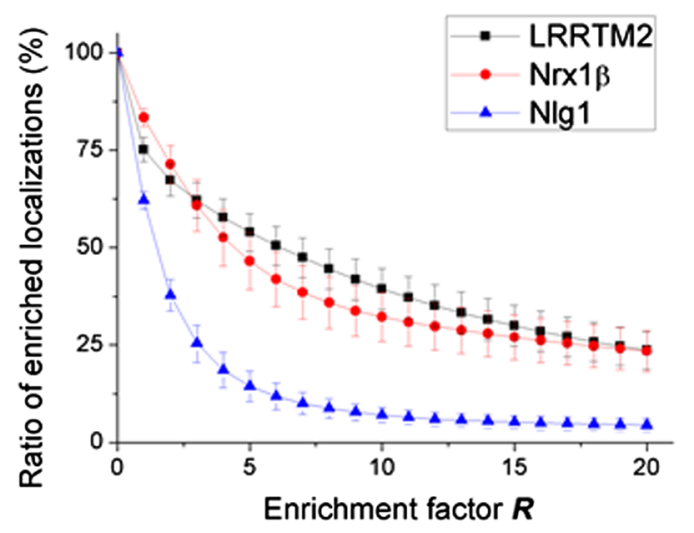

Fig. 3 The nanoscale organization of Nrx1 $\beta$ lies between that of Nlg1 and LRRTM2. (a) Color-coded images showing the enrichment factor $R$ in linear scale for Nlg1, LRRTM2 in two dendritic spines, and $\mathrm{Nrx} 1 \beta$ in a presynaptic terminal. Note the mild synaptic enrichment of Nlg1 compared to LRRTM2, which is highly concentrated in a single large domain. Nrx1 $\beta$ shows both a diffuse distribution throughout the presynapse, together with small and highly enriched nanoclusters. (b) Ratio of the enriched area relative to the whole neuronal area considered $A(R)$, as a function of the enrichment factor $R$, for the three proteins. $A(R)$ drops quickly as the enrichment factor is increased, and the curve for Nrx1 $\beta$ lies between those representing Nlg1 and LRTTM2. (c) Ratio of the number of single-molecule localizations in enriched areas $N(R)$, relative to the total number of localizations in the neuronal area, as a function of the enrichment factor $R$, for the three proteins. In this quantification, the curve for $\operatorname{Nrx} 1 \beta$ is closer to that of LRTTM2.

LRRTM $2,{ }^{18}$ we expect the homogeneously distributed $\operatorname{Nrx} 1 \beta$ and Nlg1 population to represent dynamic complexes that exhibit fast diffusion in the shaft and are transiently trapped at synapses, ${ }^{18,19}$ while the Nrx1 $\beta /$ LRRTM 2 clusters at the core of the synaptic cleft are predicted to be much more stable. This simple picture might become a little more complex if we consider that all these molecules exist as different isoforms and splice variants, e.g., Nlg1 and LRRTM2 might also interact with $\alpha$-Nrxs present at the presynapse. ${ }^{19}$

In the absence of high-quality antibodies against surface epitopes on endogenous Nrx, Nlg, or LRRTM, we monitored the distribution of recombinant AP-tagged proteins. Thus, local molecular densities should not be interpreted as absolute values quantitatively reflecting the behavior of endogenous proteins, but as relative measurements allowing comparison of the distribution of the three synaptic adhesion proteins. Nevertheless, to avoid massive overexpression of proteins that could bias their intrinsic localization, we transfected neurons through electroporation with very low deoxyribose nucleic acid (DNA) amounts. In this context, we previously quantified for Nlg1 that there was roughly one exogenous molecule for one endogenous molecule, ${ }^{18}$ and this ratio is likely to be more or less conserved for
$\operatorname{Nrx} 1 \beta$ and LRRTM2, depending on endogenous protein levels and recombinant expression vectors. Furthermore, by replacing endogenous Nlg1 by recombinant AP-tagged Nlg1 using a knock-down plus rescue strategy, we obtained a similarly synaptic distribution of $\mathrm{Nlg} 1$ by STORM, ${ }^{18}$ ruling out potential overexpression artifacts. A similar replacement approach could be used in the future for $\operatorname{Nrx} 1 \beta$ and LRRTM2. To study the nanoscale organization of those proteins, monomeric ligands, such as mSA, provide a strong advantage over divalent antibodies or multivalent streptavidin in that they do not induce cross-linking or the formation of artificial nanoclusters, which would otherwise bias the measurements. ${ }^{17,18}$

Our approach was based on the comparative statistical analysis of the distribution of individual synaptic components, imaged one at a time. However, in order to unambiguously identify the one-to-one association between presynaptic $\operatorname{Nrx} 1 \beta$ substructures and the ones corresponding to Nlg1 or LRRTM2, it would be interesting to setup a dual or triple color imaging configuration of those synaptic adhesion molecules. To this aim, one would need orthogonal labeling strategies to discriminate $\operatorname{Nrx} 1 \beta, \mathrm{Nlg} 1$, and LRRTM2. One difficulty is that $\mathrm{Nlg} 1$ and LRRTM2 do not tolerate well large extracellular or intracellular 
tags that can perturb either adhesion to $\mathrm{Nrxs},{ }^{27}$ or binding to scaffolding molecules such as PSD-95. ${ }^{28}$ Thus, one would need instead to find a strategy relying on small extracellular tags. It will also be important to localize Nrxs, Nlgs, and LRRTMs with respect to other adhesion proteins of the synaptic cleft, in particular SynCAMs ${ }^{11}$ and N-cadherin. ${ }^{29-31}$

The development of such multicolor superresolution imaging will enable us to establish a mapping of the distribution of adhesion proteins in relation to other important actors of the synapse, in particular scaffolding molecules and neurotransmitter receptors, ${ }^{9,32}$ and their potential reshaping during synaptic plasticity. For example, we previously showed that the chemical induction of long-term depression in neuronal cultures by transient application of $N$-methyl-D-aspartate (NMDA) caused a progressive disappearance of both $\mathrm{Nrx} 1 \beta$ and $\mathrm{Nlg} 1$ from the neuronal surface, ${ }^{18}$ potentially through the cleavage of $\mathrm{Nlg} 1$ extracellular domain by the protease MMP9. ${ }^{33,34}$ An extrasynaptic relocalization of SynCAM1 was also reported in response to NMDA treatment. ${ }^{11}$ It would thus be interesting to assess whether there is a similar reorganization of LRRTM2 at synapses in relationship to presynaptic Nrx1. Finally, it will be important to implement localization-based superresolution imaging methods in organotypic tissue where synaptic connectivity is preserved compared to dissociated cultures, so as to be able to apply well-established synaptic plasticity protocols. ${ }^{3,35}$

\section{Methods}

\subsection{Deoxyribose Nucleic Acid Plasmids}

The AP-Nlg1, AP-Nrx1 $\beta$, and BirA ${ }^{\mathrm{ER}}$ constructs $^{17,27}$ were kind gifts from A. Ting (MIT, Boston). AP-LRRTM2 was generated using the In-Fusion HD Cloning kit (Clontech), replacing the myc-tag from myc-LRRTM2 ${ }^{26}$ (J. de Wit, Leuwen, Belgium) by the AP-Tag (amino acid sequence GLNDIFEAQKIEWHE) as described. ${ }^{18}$ SEP-VGlut1 was a kind gift from D. Perrais (Interdisciplinary Institute for Neuroscience, Bordeaux). mSA was subcloned from the previously described pRSET-A vector generously given by S. Park (Buffalo University, New York) $)^{36,37}$ into pET-IG, a homemade vector derived from pET-24 (Novagen) and engineered to incorporate after the start codon a decahistidine tag immediately followed by a Tobacco Etch Virus cleavage site (-ENLYFQS-) and no tag on the $C$-terminus.

\subsection{Streptavidin Monomers Production and Coupling to Fluorophores}

mSA was produced as previously described. ${ }^{18}$ Briefly, mSA, encoded in the pET-IG-mSA vector, was expressed by autoinduction in Escherichia coli BL21 codon plus ${ }^{\mathrm{TM}}$ (DE3)-RIL for $12 \mathrm{~h}$ at $16^{\circ} \mathrm{C}$. Following lysis of the bacteria in denaturing conditions, the protein was purified by immobilized metal ion affinity chromatography with HIS-Buster Cobalt Affinity gel (AMOCOL) and refolded in presence of reduced and oxidized glutathione. The refolded protein was concentrated and coupled to Alexa Fluor 647 NHS ester following the recommended procedures from the manufacturer. Excess dye was removed using Sephadex G-25 medium, the conjugate was further purified to homogeneity by size exclusion chromatography, and the labeled protein was aliquoted and stored at $-80^{\circ} \mathrm{C}$ until use.

\subsection{Cell Culture and Electroporation}

Pregnant female rats were purchased weekly (Janvier Labs, Saint-Berthevin, France). Animals were handled and euthanized according to European ethical rules. Dissociated hippocampal neurons from E18 Sprague-Dawley rats embryos were prepared as described ${ }^{38}$ and electroporated with the Amaxa system (Lonza), using 500,000 cells per cuvette. The following plasmid combinations were used: (GFP or SEP-VGlut1) + BirA $^{\mathrm{ER}}+$ (AP-Nlg1, AP-LRRTM2, or AP-Nrx1 $\beta$ ) (1.5:1.5:1.5) $\mu$ g DNA. Electroporated neurons were resuspended in minimal essential medium supplemented with 10\% horse serum (MEM-HS) and plated on $18-\mathrm{mm}$ coverslips previously coated with $1 \mathrm{mg} \mathrm{ml}^{-1}$ polylysine for $2 \mathrm{~h}$, at a concentration of 50,000 cells per coverslip. Three hours after plating, coverslips were flipped onto 60-mm dishes containing a glial cell layer in neurobasal medium supplemented with $2 \mathrm{mM}$ L-glutamine and $1 \times$ NeuroCult SM1 Neuronal supplement (STEMCELL technologies), and cultured for 2 weeks at $37^{\circ} \mathrm{C}$ and $5 \% \mathrm{CO}_{2}$. Astrocytes feeder layers were prepared from the same embryos, plated between 20,000 and 40,000 cells per 60-mm dish and cultured in MEM (Fisher Scientific, Cat. No. 21090-022) containing $4.5 \mathrm{gl}^{-1}$ glucose, 2-mM L-glutamine, and $10 \%$ horse serum (Invitrogen) for 14 days.

\subsection{Direct Stochastic Optical Reconstruction Microscopy}

Primary cultured neurons expressing AP-Nlg1, AP-LRRTM2, or AP-Nrx1b were surface-labeled with a high concentration $(100 \mathrm{nM})$ of mSA-Alexa647 in Tyrode solution (15 mM D-glucose, $108 \mathrm{mM} \mathrm{NaCl}, 5 \mathrm{mM} \mathrm{KCl}, 2 \mathrm{mM} \mathrm{MgCl}$, $2 \mathrm{mM} \mathrm{CaCl}_{2}$, and $25 \mathrm{mM}$ HEPES, $\mathrm{pH}$ 7.4) containing $1 \%$ globulin-free BSA (Sigma) for $10 \mathrm{~min}$, rinsed and fixed with $4 \%$ PFA-0.2\% glutaraldehyde in PBS for $10 \mathrm{~min}$ at room temperature. Before dSTORM acquisitions, the samples were incubated in a solution of PBS containing 1:1000 fluorescent beads (Tetraspec) used as markers for image registration. dSTORM imaging of cultured neurons was performed using an inverted motorized microscope (Nikon Ti-Eclipse, Japan) equipped with a $100 \times / 1.49$ NA PL-APO objective and a perfect focus system, allowing long acquisition in oblique illumination mode. Both the ensemble and single-molecule fluorescence were collected by using a quad-band dichroic filter (Di01-R405/488/561/635, Semrock, New York). The fluorescence was collected using a sensitive EMCCD (Evolve, Photometrics, Arizona). Single-molecule localization and reconstruction were performed online with automatic feedback control of the lasers using WaveTracer module, enabling optimal single-molecule density during the acquisition. ${ }^{39}$ The acquisition and localization sequences were driven by MetaMorph software (Molecular Devices, California) in streaming mode at 50 frames/s (20-ms exposure time) using an area equal to or less than a $256 \times 256$ pixels region of interest. dSTORM superresolution images were reconstructed from 40,000 frames.

\subsection{Image Reconstruction and Analysis}

Image stacks were analyzed using a custom plugin running on MetaMorph software based on wavelet segmentation. ${ }^{39,40}$ It allows reconstructing the superresolution images by summing the positions of localized single molecules into a single image and exporting files containing the spatial coordinates of each 
localization. Fluorescent beads (Tetraspec) were used as fiduciary markers for image registration.

\subsection{Protein Quantification Using Tessellation-Based Analysis}

We used SR-Tesseler ${ }^{24}$ to quantify the molecular organization of the three synaptic adhesion proteins. Single-molecule localization coordinates were used to compute a Voronoï tessellation in order to partition the image space in polygons of various sizes centered on each localized molecule. Using this space-partitioning framework, first-rank densities $\delta_{i}^{1}$ of the molecules were computed $^{24}$ and density maps were generated by texturing the Voronoï polygons with $\delta_{i}^{1}$ values. Segmentations of the mSAlabeled AP-Nrx1 $\beta$ presynapses were performed by applying a threshold of twice the average density $\delta$ of the whole axonal region. Then subcluster organization of the $\operatorname{Nrx} 1 \beta$ distribution was obtained by applying a threshold of twice the average density of each presynapse. All selected neighboring molecules were merged together to segment presynapses and subclusters, and the size parameters were extracted by principal component analysis.

To determine the nanoscale organization of the three proteins, we first segmented the diffracted-limited low-resolution images using intensity-based thresholding in MetaMorph software. The neuronal regions $N$ were identified by segmenting the localizations inside those masks. All subsequent analyses were performed exclusively on the localizations belonging to $N$. For each protein, normalization of the density distribution was achieved by dividing the density $\delta_{i}^{1}$ of each molecule by the median $\tilde{\delta}^{1}$ of all $\delta_{i}^{1}$. The enrichment factor $R$ was defined as the ratio between the molecule density $\delta_{i}^{1}$ and the average density $\delta^{N}$ of the neuronal region. By varying $R$ (from 0 to 20), we determined enriched regions (for $R>0$ ) with a given area $A(R)$ and a number of localization $N(R) . A(R)$ reflects the surface occupation for $\delta_{i}^{1} \geq R \times \delta^{N}$ and $N(R)$ the number of localizations within this area.

\section{Acknowledgments}

We thank A. Ting and S. Park for the generous gift of DNA plasmids, B. Tessier for molecular biology, the Cell Culture Facility of the Institute (especially E. Verdier and P. Durand), and J. Gilardin for technical support. This work received funding from the Centre National de la Recherche Scientifique, Agence Nationale pour la Recherche (grants Synapse-2Dt, Nanodom, and SynAdh), Conseil Régional Aquitaine, Investissements d'Avenir Labex BRAIN, Fondation pour la Recherche Médicale, and the National Infrastructure France BioImaging (Grant No. ANR-10INBS-04-01).

\section{References}

1. S. Manley et al., "High-density mapping of single-molecule trajectories with photoactivated localization microscopy," Nat. Methods 5(2), 155157 (2008).

2. H. Shroff et al., "Live-cell photoactivated localization microscopy of nanoscale adhesion dynamics," Nat. Methods 5(5), 417-423 (2008).

3. A. Dani et al., "Superresolution imaging of chemical synapses in the brain," Neuron 68(5), 843-856 (2010).

4. M. Heilemann et al., "Subdiffraction-resolution fluorescence imaging with conventional fluorescent probes," Angew. Chemie-Int. Ed. 47(33), 6172-6176 (2008).
5. A. Sharonov and R. M. Hochstrasser, "Wide-field subdiffraction imaging by accumulated binding of diffusing probes," Proc. Natl. Acad. Sci. U. S. A. 103(50), 18911-18916 (2006).

6. G. Giannone et al., "Dynamic superresolution imaging of endogenous proteins on living cells at ultra-high density," Biophys. J. 99(4), 13031310 (2010).

7. B. Huang, H. Babcock, and X. Zhuang, "Breaking the diffraction barrier: super-resolution imaging of cells," Cell 143(7), 1047-1058 (2010).

8. A. Chazeau et al., "Nanoscale segregation of actin nucleation and elongation factors determines dendritic spine protrusion," EMBO J. 33(23), 2745-2764 (2014).

9. D. Nair et al., "Super-resolution imaging reveals that AMPA receptors inside synapses are dynamically organized in nanodomains regulated by PSD95," J. Neurosci. 33(32), 13204-13224 (2013).

10. T. Vaithianathan et al., "Nanoscale dynamics of synaptic vesicle trafficking and fusion at the presynaptic active zone," eLife 5, 1-14 (2016).

11. K. Perez de Arce et al., "Topographic mapping of the synaptic cleft into adhesive nanodomains," Neuron 88(6), 1165-1172 (2015).

12. E. Hosy, C. Butler, and J. B. Sibarita, "Organization and dynamics of AMPA receptors inside synapses-nano-organization of AMPA receptors and main synaptic scaffolding proteins revealed by super-resolution imaging," Curr. Opin. Chem. Biol. 20(1), 120-126 (2014).

13. T. C. Südhof, "Neuroligins and neurexins link synaptic function to cognitive disease," Nature 455(7215), 903-911 (2008).

14. M. A. Bemben et al., "The cellular and molecular landscape of neuroligins," Trends Neurosci. 38(8), 496-505 (2015).

15. G. J. Soler-Llavina et al., "The neurexin ligands, neuroligins and leucine-rich repeat transmembrane proteins, perform convergent and divergent synaptic functions in vivo," Proc. Natl. Acad. Sci. U. S. A. 108(40), 16502-16509 (2011).

16. K. H. Lim et al., "Stable, high-affinity streptavidin monomer for protein labeling and monovalent biotin detection," Biotechnol. Bioeng. 110(1), 57-67 (2013).

17. M. Howarth et al., "Targeting quantum dots to surface proteins in living cells with biotin ligase," Proc. Natl. Acad. Sci. U. S. A. 102(21), 75837588 (2005).

18. I. Chamma et al., "Mapping the dynamics and nanoscale organization of synaptic adhesion proteins using monomeric streptavidin," Nat. Commun. 7, 10773 (2016).

19. C. Neupert et al., "Regulated dynamic trafficking of neurexins inside and outside of synaptic terminals," J. Neurosci. 35(40), 13629-13647 (2015).

20. M. Missler et al., "Alpha-neurexins couple $\mathrm{Ca}^{2+}$ channels to synaptic vesicle exocytosis," Nature 423(6943), 939-948 (2003).

21. J. Ko et al., "LRRTM2 functions as a neurexin ligand in promoting excitatory synapse formation," Neuron 64(6), 791-798 (2009).

22. D. Comoletti et al., "Characterization of the interaction of a recombinant soluble neuroligin-1 with neurexin-1 $\beta$," J. Biol. Chem. 278(50), 5049750505 (2003).

23. T. J. Siddiqui et al., "LRRTMs and neuroligins bind neurexins with a differential code to cooperate in glutamate synapse development," J. Neurosci. 30(22), 7495-7506 (2010).

24. F. Levet et al., "SR-Tesseler: a method to segment and quantify localization-based super-resolution microscopy data," Nat. Methods 12(11), 1065-1071 (2015).

25. D. S. Liu et al., "Quantum dot targeting with lipoic acid ligase and halotag for single-molecule imaging on living cells," ACS Nano 6(12), 11080-11087 (2012).

26. J. de Wit et al., "LRRTM 2 interacts with neurexin 1 and regulates excitatory synapse formation," Neuron 64(6), 799-806 (2009).

27. D. S. Liu et al., "Imaging trans-cellular neurexin-neuroligin interactions by enzymatic probe ligation," Plos One 8(2), e52823 (2013).

28. G. Giannone et al., "Neurexin- $1 \beta$ binding to neuroligin-1 triggers the preferential recruitment of PSD-95 versus gephyrin through tyrosine phosphorylation of neuroligin-1," Cell Rep. 3(6), 1996-2007 (2013).

29. N. Uchida et al., "The catenin/cadherin adhesion system is localized in synaptic junctions bordering transmitter release zones," J. Cell Biol. 135(3), 767-779 (1996).

30. P. Mendez et al., "N-cadherin mediates plasticity-induced long-term spine stabilization," J. Cell Biol. 189(3), 589-600 (2010).

31. O. Bozdagi et al., "Increasing numbers of synaptic puncta during latephase LTP: N-cadherin is synthesized, recruited to synaptic sites, and required for potentiation," Neuron 28(1), 245-259 (2000). 
32. H. D. MacGillavry et al., "Nanoscale scaffolding domains within the postsynaptic density concentrate synaptic AMPA receptors," Neuron 78(4), 615-622 (2013).

33. R. T. Peixoto et al., "Transsynaptic signaling by activity-dependent cleavage of neuroligin-1," Neuron 76(2), 396-409.(2012).

34. K. Suzuki et al., "Activity-dependent proteolytic cleavage of neuroligin1," Neuron 76(2), 410-422 (2012).

35. R. Galland et al., "3D high- and super-resolution imaging using singleobjective SPIM," Nat. Methods 12(7), 641-644 (2015).

36. K. H. Lim et al., "Engineered streptavidin monomer and dimer with improved stability and function," Biochemistry 50(40), 8682-8691 (2011).

37. D. Demonte et al., "Structure-based engineering of streptavidin monomer with a reduced biotin dissociation rate," Proteins 81(9), 1621-1633 (2013).

38. S. Kaech and G. Banker, "Culturing hippocampal neurons," Nat. Protocol 1(5), 2406-2415 (2006).

39. A. Kechkar et al., "Real-time analysis and visualization for singlemolecule based super-resolution microscopy," Plos One 8(4), e62918 (2013).

40. I. Izeddin et al., "Wavelet analysis for single molecule localization microscopy," Opt. Express 20, 2081-2095 (2012).

Ingrid Chamma is a postdoctoral fellow with the team of Olivier Thoumine in Bordeaux, France. She studied genetics and neuroscience at the University of Paris Diderot and received her PhD from the University Pierre and Marie Curie, Paris. Her current research focuses on the development of new molecular tools and their application to superresolution microscopy for protein targeting in complex microenvironments.
Florian Levet studied computer sciences in Bordeaux, France. After a $\mathrm{PhD}$ at the LaBRI, he worked as a research engineer at the Bordeaux Imaging Center. He then joined the group of Jean-Baptiste Sibarita at the Interdisciplinary Institute for Neuroscience (IINS). His current research focuses on the development of new quantification and visualization methods for single-molecule localization microscopy, with a strong interest in geometric tessellations.

Jean-Baptiste Sibarita has a PhD thesis in physics and is expert in live cell microscopy and image processing and analysis. He has coheaded and developed the Cellular and Tissular Imaging Platform of the Institut Curie, Paris, for 12 years. Since 2009, he started his own group "Quantitative Imaging of the Cell" in the newly created Interdisciplinary Institute of Neuroscience, Bordeaux.

Matthieu Sainlos is a senior researcher at the French National Center for Scientific Research working at the IINS, Bordeaux, in the group of Daniel Choquet. His current research is focused on the use of multidisciplinary approaches from chemistry and protein engineering to imaging in order to develop tools and methods to investigate the molecular mechanisms of synaptic proteins. The strategies he develops include modulating protein interactions and monitoring specific proteins complexes.

Olivier Thoumine is team leader at IINS, Bordeaux. He is a biophysicist with a strong expertise in the mechanisms of cell adhesion. He has been using a combination of computer simulations, single-molecule imaging, and biomimetic assays to characterize molecular clutch mechanisms between the actin flow and $\mathrm{N}$-cadherin adhesions in growth cones and dendritic filopodia, and to decipher the role of neurexin/neuroligin complexes in synapse differentiation and function. 